\title{
ARTICLE
}

\section{Biochemical and hematological profile of Otaria flavescens in the reproductive colony of Cobquecura, central-south Chile}

Perfil bioquímico y hematológico de Otaria flavescens en la colonia reproductiva de Cobquecura en Chile centro-sur

\section{Rodrigo R. González-Saldía ${ }^{1,4}$, Paulina A. Lincoñir ${ }^{4}$, Renato A. Quiñones ${ }^{2,3}$, M. Cristina Krautz ${ }^{2}$, Sam Catchpole ${ }^{2}$ and Wilfredo Alarcón ${ }^{2}$}

${ }^{1}$ Unidad de Biotecnología Marina, Facultad de Ciencias Naturales y Oceanográficas, Universidad de Concepción, Casilla 160-C, Concepción, Chile

${ }^{2}$ Programa de Investigación Marina de Excelencia (PIMEX), Departamento de Oceanografía, Facultad de Ciencias Naturales y Oceanográficas Universidad de Concepción, Casilla 160-C, Concepción, Chile

${ }^{3}$ Interdisciplinary Center for Aquaculture Research (INCAR), Universidad de Concepción, O’Higgins 1695, Concepción, Chile ${ }^{4}$ Centro de Investigación Oceanográfica del Pacífico Sur-Oriental (FONDAP-COPAS), COPAS Sur Austral, Universidad de Concepción, Casilla 160-C, Concepción, Chile.rogonzal@udec.cl

\begin{abstract}
Resumen.- A pesar de la importancia ecológica del lobo marino común Otaria flavescens, existe escasa información referente a su química sanguínea y hematología, los cuales pueden ser utilizados para evaluar el estado de salud de las poblaciones. El presente trabajo, reporta por primera vez la determinación de 14 parámetros químicos sanguíneos de 0 . flavescens y su fórmula leucocitaria obtenida desde 15 individuos pertenecientes a una colonia reproductiva localizada en Cobquecura, zona centro-sur de Chile, durante 2 muestreos realizados, 2009 y 2011. Los resultados indican que existe una alta regularidad bioquímica para los parámetros estudiados. No obstante, se presentan diferencias significativas en la concentración plasmática de calcio entre ambos sexos y entre las concentraciones promedio de proteínas totales, globulinas, colesterol y actividad de colinesterasa de los 2 muestreos estudiados. Comparado con otros perfiles bioquímicos reportados para mamíferos marinos, en esta especie se determinó una alta concentración de globulinas y capacidad inhibitoria de antiproteasa (cercana al 100\%), una moderada a baja actividad de colinesterasa y una concentración de glucosa en el rango de lo ya reportado. Finalmente, la fórmula leucocitaria muestra una eosinófilia moderada a alta para el primer muestreo analizado (8,3-17,6\%), lo que sugiere la posible presencia de alguna parasitosis en la población estudiada. La presente información puede ser útil para la determinación del estado de salud de poblaciones de 0 . flavescens, y por lo tanto, podría contribuir a la conservación de la especie en el Pacífico Sur Oriental.
\end{abstract}

Palabras clave: Otaria flavescens, lobo marino común, química sanguínea, hematología, fórmula leucocitaria

Abstract.- Despite the ecological importance of the common sea lion Otaria flavescens, there is currently no information available on the blood chemistry and hematology of this species. Here, we report for the first time, 14 blood chemistry variables of 0 . flavescens and differential blood cell counts obtained from 15 resident individuals from a reproductive colony located in Cobquecura on the coast of central-south Chile, during 2 sampling periods, in 2009 and 2011. There was a high degree of biochemical regularity in studied parameters, although the plasmatic concentration of calcium differed significantly between genders; average concentrations of total proteins, globulins, cholesterol, and cholinesterase activity varied significantly between sampling periods. In comparison with the biochemical profiles reported for other marine mammals, 0. flavescens had greater concentrations of globulins; an inhibitory capacity of antiproteases (close to 100\%); and moderate to low cholinesterase activity and similar concentrations of glucose. Finally, differential blood cells counts indicated moderate to high eosinophilia (8.3-17.6\%) in individuals sampled during the first sampling period, which suggests the possible presence of parasitism within this population. These results could be useful for assessing the state of health of $\mathrm{O}$. flavescens populations and, therefore, contribute to species conservation in the Southeast Pacific.

Key words: Otaria flavescens, South American sea lion, blood chemistry, hematology, differential leukocyte counts 


\section{INTRODUCTION}

The South American sea lion, Otaria flavescens (Shaw, 1800 ), is one of the 5 species belonging to the subfamily Otariinae (family Otariidae). It is the only genera of the Otaria (Riedman 1990, Cappozzo \& Perrin 2009). Its spatial distribution includes the coasts of South America, with relatively abundant populations from northern Peru (Scheffer 1958, Riedman 1990) to the Diego Ramírez Islands in the Austral Pacific off Chile (Osgood 1943, Schlatter \& Riveros 1997). Along the Atlantic coast, this species is distributed from the south of Torres in Brazil (Rosas et al. 1994) to Tierra del Fuego in Argentina (King 1983, Dans et al. 2012), as well as the Falkland/Malvinas and Isla de los Estados (Osgood 1943, Bastida \& Rodríguez 2003). Typically piscivorous (Vaz-Ferreira 1982, Crespo et al. 1997), this species has a varied diet including crabs, cephalopods (mollusks), squid and gastropods (Aguayo \& Maturana 1973, George-Nascimento et al. 1985, Sielfeld et al. 1997, Aguayo-Lobo et al. 1998, Hückstädt \& Antezana 2006, Hückstädt et al. 2007, De la Torriente et al. 2010, Muñoz et al. 2013). However, the main dietary items of these animals are largely determined by the prey species available in their habitat, making them opportunistic predators (Sielfeld et al. 1997, Koen-Alonso et al. 2000).

Chile hosts the greatest abundance of South American sea lions in the world, with a population of approximately 150,000 individuals (Sielfeld et al. 1997, Aguayo-Lobo et al. 1998, Oporto et al. 1999, Venegas et al. 2001, Sepúlveda et al. 2007, Bartheld et al. 2008, Oliva et al. 2012). While overall population estimates have increased steadily over the past decade, certain populations appear to be affected by marine pollution and a decrease in prey abundance due to the overexploitation of fisheries (Venegas et al. 2001, Sepúlveda et al. 2011).

Previous research efforts on this species have focused on determining abundance and population distribution (e.g., Sielfeld et al. 1997, Aguayo-Lobo et al. 1998, Bartheld et al. 2008, Sepúlveda et al. 2011). However, there are scarce data on blood biochemistry and hematology (Ikehara et al. 1996). The assessment of blood chemistry and hematology profiles allows the determination of analytes that indicate the state of health and development of these animals (Roletto 1993), taking into account various physiological processes (Panfoli et al. 1999, Lehninger et al. 2001, Yuan et al. 2001). These profiles vary with the sex, developmental stage, and physical condition of an individual (Urich 1994, Sterner \& Elser 2002).
In this study, we report for the first time 14 blood chemistry parameters and hemograms obtained from 15 O. flavescens individuals belonging to the breeding colony at Cobquecura. This is the most important breeding colony of the central zone of Chile, with approximately 2,500 individuals (Sepúlveda et al. 2011).

\section{Materials AND METHODS}

Fifteen sea lions between 115 to $165 \mathrm{~cm}$ length, including 10 juveniles, four sub-adult males, and one adult female, were sampled during July $2009(n=6)$ and April $2011(n=$ 9) from the coastal area of the Nature Sanctuary of Islote Lobería de Cobquecura (360.7'53”'S-72 48'29”W), which has been state protected since 1992, within the Biobío Region of central southern Chile (Fig. 1).

During the sardine fishery catch, sea lions take advantage of the aggregation of small pelagic driven by purse seine nets to feed freely. This provides an ideal opportunity to catch individuals for sampling. Individuals were captured with a lasso in the water and immediately transferred to a mesh net on board a small purse seine vessel belonging to the sardine fishing fleet, just outside the waters of the Nature Sanctuary. Animals immobilized in the net were then transferred to a small artisan fishing vessel ( $8 \mathrm{~m}$ length) and taken to land. Capture times were never more than 20 min per animal, from first contact with the lasso to securing the animal in the small boat. The time elapsed between securing the animal in the boat and reaching land was approximately $15 \mathrm{~min}$. During the procedure, animals were monitored by a veterinarian, and only apparently healthy animals were selected for sampling; we considered healthy animals to be those which were found without wounds and with sufficient strength to resist capture. On land, animals were anaesthetized using isoflurane gas $(0.5$ a $2.5 \%)$ with oxygen, administered using a conical mask and a portable vaporizer (Gales \& Mattlin 1998). Once the animals were anaesthetized, they were weighed using a stretcher and tripod attached to a digital scale $( \pm 0.1 \mathrm{~kg})$ and total length was determined with a flexible measuring tape from the tip of the nose to the tip of the tail (standard length, $\mathrm{cm}$ ). Given logistical difficulties during the second sampling campaign, it was not possible to obtain the body weights of all individuals.

Blood samples were taken via puncture of the caudal gluteal vein with a heparinized syringe. Afterwards, animals were allowed to recover from anesthesia while being monitored, and were then released a few meters 


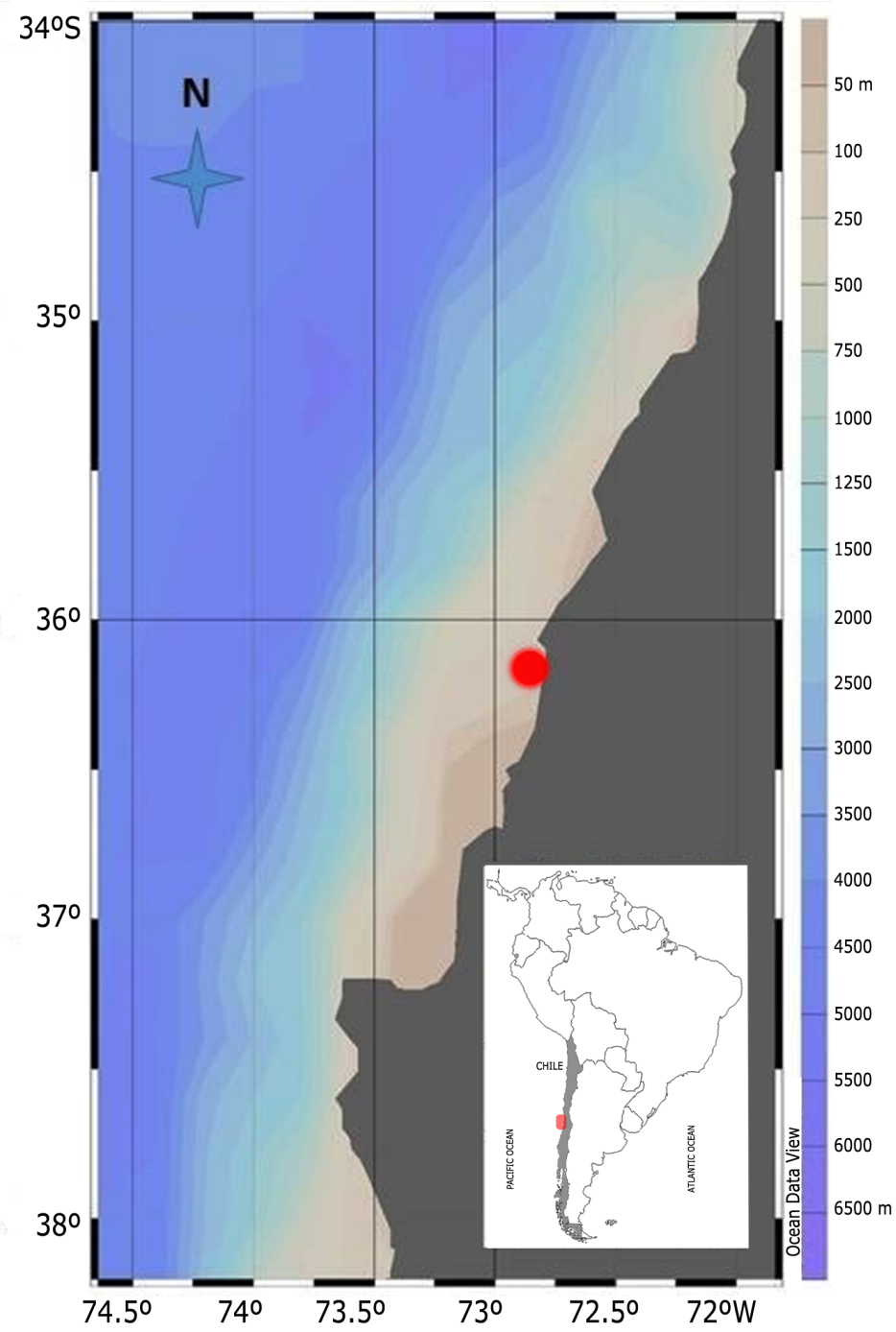

Figure 1. Study area, with the Nature Sanctuary of Islote Lobería de Cobquecura marked by a circle / Zona muestreo Santuario de la Naturaleza Islote Lobería de Cobquecura, señalada con un círculo from the sea. All animals survived this sampling procedure. Blood samples were immediately centrifuged at $1000 \mathrm{x} g \mathrm{x}$ $10 \mathrm{~min}\left(4^{\circ} \mathrm{C}\right)$ to separate the plasma and the formal elements in the blood, and plasma samples were stored at $-20^{\circ} \mathrm{C}$ until analysis.

\section{BLOOD CHEMISTRY ANALYSIS}

Biochemical profiles included albumin, total protein, phosphorus, calcium, lactate, glucose, cholesterol, urea, cholinesterase, lactate dehydrogenase, alanine aminotransferase (ALT), aspartate transaminase (AST), lysozyme and inhibitory activity of antiprotease were analyzed using a combination of methodologies.
The analysis of albumin, total proteins, phosphorus, calcium, glucose, cholesterol, urea, ALT and AST were determined according to the protocol of clinical analysis based on kits by the company Human Diagnostics (Wiesbaden, Germany). The activity of cholinesterase (ChE) and lactate were determined according to the clinical analysis kits protocol established by the company Sentinel Diagnostics (Milan, Italy).

The activity of the catabolic lactate dehydrogenase enzyme (LDH) was measured using a modified reaction mix from the Schiedek (1997) method, which contained a buffer of $\mathrm{K}_{2} \mathrm{HPO}_{4}$, NADH and pyruvate for LDH. Reactions were initiated with the addition of an aliquot of 
the supernatant, and the decay in NADH absorption was measured to $340 \mathrm{~nm}$. Enzymatic activity was corrected by the unspecific oxidation of NADH.

Lysozyme activity was determined using a method based on the lysis of Micrococcus lysodeikticus, according to Ellis (1990a). A unit (U) of lysozyme was defined as the quantity of enzyme necessary to cause a decrease in the absorbance by 0.001 units per min. Lysozyme activity was standardized to $\mathrm{U} \mathrm{mL}^{-1}$ plasma. A standard egg lysozyme calibration curve was constructed, which allowed transforming the data to concentrations of lysozyme per unit volume of plasma $\left(\mu \mathrm{g} \mathrm{mL}^{-1}\right)$.

The antiprotease activity of plasma (capacity of trypsin inhibition by alfa-2-macroglobulin) was quantified using a modified essay by Ellis (1990b) and Zuo \& Woo (1997), which is based on the ability of the antiprotease of the study organism to inhibit the activity of trypsin. The inhibitory capacity of trypsin was expressed as the percentage difference between the absorbance of the control (without plasma, without inhibition) minus the absorbance of the sample (which contains antiprotease activity), divided by the absorbance of the control.

\section{Hematology}

In parallel to blood chemistry analyses, blood smears were made to determine the differential blood count. Smears were dyed with May-Grunwald-Giemsa. Differential blood cells (i.e., monocytes, eosinophils, lymphocytes, polymorphonuclears, leukocyte bacilliforms and basophils) were counted using an optical microscope with 40X magnification.

\section{Statistical analyses}

Considering the small sample size, we decided to use the non-parametric Mann-Whitney test (IBM SPSS Statistics 20) to determine whether the blood variables differ significantly between the first and second sampling periods, as well as between genders $(P<0.05)$.

\section{Results}

\section{BLOOD CHEMISTRY ANALYSIS}

Standard length of sea lions ( $\mathrm{n}=11$ males and 4 females) ranged between $115 \mathrm{~cm}$ and $165 \mathrm{~cm}$, and mass ranged between $49 \mathrm{~kg}$ and $116.2 \mathrm{~kg}$. No differences in blood chemistry parameters were detected between genders, with the exception of calcium, which was lower in females than in males $\left(7.8 \pm 1.1\right.$ and $10.4 \pm 2.0 \mathrm{mg} \mathrm{dL}^{-1}$, respectively; $P<0.05)$ considering both samplings dates.

The data presented in Table 1 shows mean values \pm $\mathrm{SD}$, and also includes the data range for each of the blood chemistry parameters analyzed during each sampling period (2009 and 2011). During the first sampling period, significantly higher concentrations were found for total proteins, globulins, and cholesterol; thus, the activity of
Table 1. Mean values, standard deviation and data ranges of blood chemistry of the South American sea lion, Otaria flavescens, obtained in the coastal area of the Nature Sanctuary of Islote Lobería de Cobquecura $\left(36^{\circ} 0.7^{\prime} 53^{\prime \prime} \mathrm{S}-72^{\circ} 48^{\prime} 29^{\prime \prime} \mathrm{W}\right)$. The samplings were made in July $2009(n=6)$ and April $2011(n=9)$ / Valores promedio, desviación estándar y rango de datos para química sanguínea de lobo marino Sur Americano Otaria flavescens recolectados en la costa del área del Santuario de la Naturaleza del Islote Lobería de Cobquecura $\left(36^{\circ} 0,7^{\prime} 53^{\prime \prime} \mathrm{S}-72^{\circ} 48^{\prime} 29^{\prime \prime} \mathrm{W}\right)$. Los muestreos fueron realizados en julio $2009(n=6)$ y abril $2011(n=9)$

\begin{tabular}{|c|c|c|c|c|}
\hline Parameters & Sampling 1 & $\begin{array}{l}\text { Data Range } \\
\text { Sampling } 1\end{array}$ & Sampling 2 & $\begin{array}{l}\text { Data Range } \\
\text { Sampling } 2\end{array}$ \\
\hline $\operatorname{Albumin}\left(\mathrm{g} \mathrm{dL}^{-1}\right)$ & $4.3 \pm 2.4$ & $1.8-7.9$ & $4.1 \pm 1.1$ & $1.6-5.4$ \\
\hline Total proteins $\left(\mathrm{g} \mathrm{dL}^{-1}\right)$ & $25.5 \pm 8.3^{*}$ & $14.5-34.3$ & $8.1 \pm 2.1$ & $4.1-10.8$ \\
\hline Globulins $\left(\mathrm{g} \mathrm{dL}^{-1}\right)$ & $21.2 \pm 9.3^{*}$ & $9.4-31.4$ & $4.1 \pm 1.3$ & $1.8-5.6$ \\
\hline Phosphorus (mg dL $L^{-1}$ ) & $10.7 \pm 7.2$ & $4.7-23.9$ & $5.2 \pm 1.4$ & $2.9-7.3$ \\
\hline Calcium $\left(\mathrm{mg} \mathrm{dL}^{-1}\right)$ & $12.5 \pm 3.3$ & $8.7-17.8$ & $8.6 \pm 1.1$ & $6.7-10.2$ \\
\hline Lactate $\left(\mathrm{mg} \mathrm{dL}^{-1}\right)$ & $71.7 \pm 15.7$ & $54.6-95.5$ & $36.7 \pm 15.8$ & $18.4-70.7$ \\
\hline Glucose $\left(\mathrm{mg} \mathrm{dL}^{-1}\right)$ & $145.7 \pm 23.9$ & $117.0-173.6$ & $119.4 \pm 24.4$ & $84.9-157.3$ \\
\hline Cholesterol (mg dL $\left.\mathrm{m}^{-1}\right)$ & $967.9 \pm 393.9^{*}$ & $577.3-1552.5$ & $223.1 \pm 70.3$ & $132.3-262.8$ \\
\hline Cholinesterase (UI L ${ }^{-1}$ ) & $441.3 \pm 182.3^{*}$ & $131.6-592.2$ & $815.6 \pm 125.6$ & $592.2-954.1$ \\
\hline LDH (IU L $\left.{ }^{-1}\right)$ & $280 \pm 0.1$ & $155.0-412.6$ & n.d. & n.d. \\
\hline Lysozyme (IU mL $\left.{ }^{-1}\right)$ & $119.1 \pm 65.4$ & $50.0-180.0$ & n.d. & n.d. \\
\hline Inhibitory Antiprotease Activity (\%) & $96.6 \pm 2.3$ & $94.4-99.0$ & n.d. & n.d. \\
\hline Urea $\left(\mathrm{mg} \mathrm{dL}^{-1}\right)$ & $90.4 \pm 9.9$ & $86.3-100.2$ & $94.1 \pm 24.5$ & $66.8-131.4$ \\
\hline AST (IU L L $)$ & $19.1 \pm 6.1$ & $13.1-24.4$ & $21.3 \pm 12.8$ & $7.9-49.1$ \\
\hline ALT $\left(\mathrm{IU} \mathrm{L}^{-1}\right)$ & $22.4 \pm 7.6$ & $14.7-32.3$ & $10.2 \pm 6.9$ & $0.9-20.1$ \\
\hline AST/ALT & $1.0 \pm 0.6$ & $0.5-1.7$ & $7.8 \pm 12.7$ & $0.5-32.0$ \\
\hline
\end{tabular}

n.d. $=$ no data $* P<0.05$ 
the cholinesterase was found to be less than during the second sampling period (Table 1). Also, during the first sampling period, a high inhibitory capacity of antiprotease was recorded, almost $100 \%$, although this parameter, as well as LDH and lysozyme, was not determined during the second sampling period.

An interesting finding was the ratio between cholesterol/cholinesterase relative to the body length of individuals, where a constant value of this independent length ratio was observed, with the exception of one individual, which lay absolutely outside the range due to its low cholinesterase activity (Fig. 2).

\section{Hematology}

The leucocyte range obtained for this species during both sampling periods is presented in Table 2. The most abundant circulating cells in the blood of this species were polymorphonuclears (PMN) and lymphocytes. Significant differences were observed $(P<0.05)$ between the first and second sampling periods for eosinophils, polymorphonuclears, monocytes, and basophils.

All individuals analyzed during the first sampling period had moderate to high eosinophilia (range $=8.3$ to $17.6 \%$ ). In contrast, during the second sampling period, individuals presented a low to moderate percentage of eosinophils, between 2.5 and $9.9 \%$.

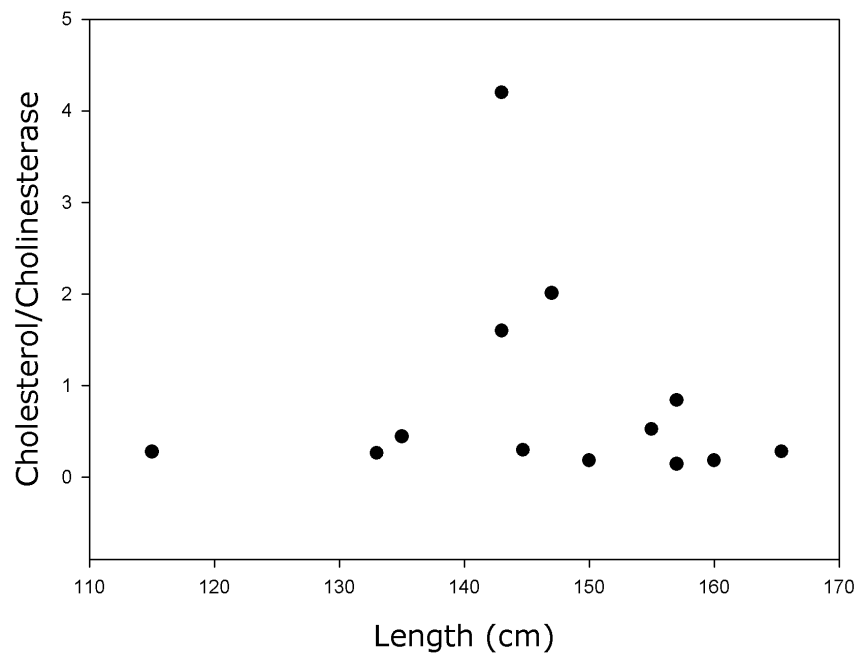

Figure 2. Relationship between body length of individuals and the cholesterol/cholinesterase activity ratio / Relación entre longitud de los individuos y la razón colesterol/actividad de colinesterasa

Table 2. Mean values, standard deviation and data range of differential blood cell counts of the South American sea lion Otaria flavescens collected in the coastal area of the Nature Sanctuary of Islote Lobería de Cobquecura $\left(36^{\circ} 0.7^{\prime} 53^{\prime \prime} \mathrm{S}-72^{\circ} 48^{\prime} 29^{\prime \prime} \mathrm{W}\right)$. Sampling was carried out in July $2009(n=6)$ and April $2011(n=9)$ / Valores promedio, desviación estándar y rango de datos para la fórmula leucocitaria de lobo marino Sur Americano Otaria flavescens recolectados en la costa del área del Santuario de la Naturaleza del Islote Lobería de Cobquecura $\left(36^{\circ} 0.7^{\prime} 53^{\prime \prime} \mathrm{S}-72^{\circ} 48^{\prime} 29^{\prime \prime} \mathrm{W}\right)$. Los muestreos fueron realizados en julio $2009(n=6)$ y abril $2011(n=9)$

\begin{tabular}{lcccc}
\hline Parameter & Sampling 1 & $\begin{array}{c}\text { Data Range } \\
\text { Sampling 1 }\end{array}$ & Sampling 2 & $\begin{array}{c}\text { Data Range } \\
\text { Sampling 2 }\end{array}$ \\
\hline Polymorphonuclear (\%) & $45.8 \pm 2.1$ & $41.4-50.8$ & $53.0 \pm 4.2^{*}$ & $46.6-59.6$ \\
Lymphocyte (\%) & $32.9 \pm 2.4$ & $24.6-41.3$ & $36.0 \pm 5.0$ & $30.1-42.7$ \\
Eosinophil (\%) & $11.9 \pm 3.3^{*}$ & $8.3-17.6$ & $6.5 \pm 2.3$ & $2.5-9.9$ \\
Monocyte (\%) & $8.7 \pm 1.0^{*}$ & $6.4-12.4$ & $3.2 \pm 1.0$ & $1.1-4.7$ \\
Leukocytebacilliforms (\%) & $0.6 \pm 0.3$ & $0.3-0.7$ & $0.2 \pm 0.5$ & $0.0-1.5$ \\
Basophil (\%) & $0.2 \pm 0.3$ & $0.0-0.4$ & $0.8 \pm 0.4^{*}$ & $0.3-1.4$ \\
\hline
\end{tabular}

$* P<0.05$ 


\section{Discussion}

To the best of our knowledge there has been no published information regarding the blood chemistry and differential blood cell counts for Otaria flavescens prior to this study. For the most part, our results revealed biochemical consistencies between genders and sampling periods (Table 1). Although calcium differed between females and males in this study, the values were still within ranges reported for other marine mammals (Table 3), including otariids. Average values obtained for albumin and phosphorus were also similar to those reported in the literature (Table 3). These values were also consistent with those described by Roletto (1993) for rehabilitated, clinically healthy California sea lions (Zalophus californianus), northern elephant seals (Mirounga angustirostris), and harbor seals (Phoca vitulina richardii), as well as with the ranges described in Bossart et al. (2001) for these analytes.

In wild marine mammals, the concentration of glucose in the blood can vary due to age, diet, physical activity, metabolism, environmental conditions, and seasonal variation (Halloran \& Pearson 1972, LeResche et al. 1974, Seal et al. 1975, Matula et al. 1980, Colares et al. 2000). In addition, a glucose increase in plasma can be the product of stress following the manipulation of animals during sampling (Seal et al. 1975, LeResche et al. 1974, Hyvarinen et al. 1975, Koopman et al. 1995). In the present study, glucose concentration was on average higher during both study periods (Tables 1 and 3 ), in comparison with the California sea lion, which is the otariid species most closely related to $O$. flavescens with available data (Table 3 ). The values of glucose obtained for $O$. flavescens were similar to those observed in seals and elephant seals (Davis et al. 1991, Trumble \& Castellini 2002, Roletto 1993, Table 3), as well as to the Steller sea lion (Eumetopias jubatus; Lander et al. 2014).

Davis (1983) and Davis et al. (1991) found lactate values of captive harbor seals that were considerably lower than those reported in this study; however, the lactate values reported by these authors (Table 3 ) were based on measurements of individuals in a resting state. The elevated values of lactate found for Otaria may represent the period of post-immersion recovery of studied individuals, as found for other vertebrates (Hochachka \& Somero 1973). Accumulated plasma lactate concentrations in studied individuals (Table 1), when transformed to molar concentrations, are within the range of 4.1 to $7.9 \mathrm{mM}$. The values were also one order of magnitude greater than reported plasma lactate for the post-diving recovery period $(0.55 \mathrm{mM})$ determined for Weddell seals (Butler \& Jones 1997). However, studies conducted by Werner \& Campagna (1995), determined that the maximum dive time for $O$. flavescens corresponds to $7.7 \mathrm{~min}$, but these authors did not report the plasma lactate concentration after these dives. It is interesting to note that Weddell seals have longer dive durations than O. flavescens, and accumulate less plasma lactate determined in this study. This might imply that sea lions reach higher lactate concentrations more quickly compared to Weddell seals. It was therefore not possible to infer the relationship between the plasma concentrations of this analyte determined in the present study with the dive time observed by Werner \& Campagna (1995).

The high value of total proteins from the first sampling period compared with the second sampling period was noteworthy (Table 1). Values from the second period were comparable with values in the literature (Table 3 ), suggesting an altered physiological state for individuals from the first sampling period, given that total proteins were at least twice as high as those reported for other marine mammals (Table 3). However, the albumin values obtained during both sampling periods were within the same range as those in the literature (Table 3). Given this observation, it appears that globulin proteins contributed to the increase in protein fraction in these animals during the first sampling period. This increase can be explained by the high concentration of lipoproteins necessary to stabilize high values of cholesterol registered from the first sampling period (Table 1), close to the reported lipid range for phocids (1200 to $3000 \mathrm{mg} \mathrm{dL}^{-1}$; Dangerfield et al. 1976). However, cholesterol values of $O$. flavescens were greater than those reported for other pinnipeds (Table 3). Values from the second sampling period were lower and within the range of values reported in the literature (Table 3) and displayed significant differences $(P<0.05)$ compared to the first sampling period. A possible explanation is that the principal prey of $O$. flavescens is the anchovy (Engraulis ringens), which have a more carnivorous diet during the austral winter (July 2009) compared with the end of the austral summer (April 2011), when it is feeds almost exclusively on phytoplankton (Krautz et al. 2012).

The innate immune response is the first line of defense against a broad spectrum of pathogens in the environment and an important physiological mechanism for maintaining homeostasis. Lysozyme is an important molecule of the innate immune response, which mediates protection 
Table 3. Average values and standard deviations were obtained for each of the blood chemistry values and differential blood cell counts in Otaria flavescens, compared with existing information in the literature on pinnipeds and other marine mammals. Parameter values are listed in ascending order / Valores promedios y desviación estándar obtenidos para cada uno de los parámetros de química sanguínea y fórmula leucocitaria determinado en Otaria flavescens comparada con la información existente en la literatura respecto a pinnípedos y otros mamíferos marinos. Los valores de los parámetros están presentados en orden ascendente

\begin{tabular}{|c|c|c|c|}
\hline Parameter & Species & Value & Reference \\
\hline \multirow[t]{12}{*}{ Albumin $\left(\mathrm{g} \mathrm{dL}^{-1}\right)$} & Gray whale/Eschrichtius robustus & $1.8 \pm$ n.d. & Dailey et al. 2000 \\
\hline & Walrus/Odobenus rosmarus & $2.2-3.1$ & Bossart et al. 2001 \\
\hline & Harbor seal/Phoca vitulina & $2.5-3.3$ & Bossart et al. 2001 \\
\hline & California Sea Lions/Zalophus californianus & $2.7-3.7$ & Bossart et al. 2001 \\
\hline & Northern elephant seal/Mirounga angustirostris & $3.2-3.9$ & Bossart et al. 2001 \\
\hline & Harbor seal/Phoca vitulina & $3.39 \pm 0.41$ & Trumble \& Castellini. 2002 \\
\hline & Amazonian manatee/Trichechus inunguis & $3.4 \pm 0.1$ & Colares et al. 2000 \\
\hline & Steller sea lion/Eumetopias jubatus & $3.5-4.5$ & Bossart et al. 2001 \\
\hline & California Sea Lions/Zalophus californianus & $3.8 \pm 0.2$ & Roletto 1993 \\
\hline & Pacific Harbour Seal/Phoca vitulina richardii & $3.8 \pm 0.2$ & Roletto 1993 \\
\hline & Northern elephant seal/Mirounga angustirostris & $3.8 \pm 0.4$ & Roletto 1993 \\
\hline & South American sea lion/Otaria flavescens (July 2009-April 2011) & $4.2 \pm 1.6$ & This study \\
\hline \multirow[t]{8}{*}{ Total protein $\left(\mathrm{g} \mathrm{dL}^{-1}\right)$} & Harbor seal/Phoca vitulina & $0.66 \pm 0.08$ & Trumble \& Castellini 2002 \\
\hline & Gray whale/Eschrichtius robustus & $5.7 \pm$ n.d. & Dailey et al. 2000 \\
\hline & Amazonian manatee/Trichechus inunguis & $6.4 \pm 0.1$ & Colares et al. 2000 \\
\hline & Northern elephant seal/Mirounga angustirostris & $7.2 \pm 1.0$ & Roletto 1993 \\
\hline & Pacific Harbour Seal/Phoca vitulina richardii & $7.3 \pm 0.8$ & Roletto 1993 \\
\hline & California Sea Lion/Zalophus californianus & $8.0 \pm 1.0$ & Roletto 1993 \\
\hline & Juan Fernández fur seal/Arctocephalus philippi & $9.4 \pm 1.7$ & Sepúlveda et al. 1999 \\
\hline & South American sea lion/Otaria flavescens (July 2009-April 2011) & $12.6 \pm 8.3$ & This study \\
\hline \multirow[t]{4}{*}{ Globulin $\left(\mathrm{g} \mathrm{dL}^{-1}\right)$} & Harbor seal/Phoca vitulina & $0.32 \pm 0.05$ & Trumble \& Castellini 2002 \\
\hline & Amazonian manatee/Trichechus inunguis & $3.0 \pm 0.1$ & Colares et al. 2000 \\
\hline & Gray whale/Eschrichtius robustus & $3.9 \pm$ n.d. & Dailey et al. 2000 \\
\hline & South American sea lion/Otaria flavescens (July 2009-April 2011) & $10.9 \pm 10.4$ & This study \\
\hline \multirow[t]{8}{*}{ Phosphorus (mg dL ${ }^{-1}$ ) } & California Sea Lions/Zalophus californianus & $1.8-7.8$ & Bossart et al. 2001 \\
\hline & Harbor seal/Phoca vitulina & $1.9-7.4$ & Bossart et al. 2001 \\
\hline & Gray whale/Eschrichtius robustus & $4.1 \pm$ n.d. & Dailey et al. 2000 \\
\hline & Walrus/Odobenus rosmarus & $4.4-6.4$ & Bossart et al. 2001 \\
\hline & Steller sea lion/Eumetopias jubatus & $5.3-9.1$ & Bossart et al. 2001 \\
\hline & Northern elephant seal/Mirounga angustirostris & $6.6-9.9$ & Bossart et al. 2001 \\
\hline & Harbor seal/Phoca vitulina & $6.71 \pm 1.56$ & Trumble \& Castellini 2002 \\
\hline & South American sea lion/Otaria flavescens (July 2009-April 2011) & $7.4 \pm 5.2$ & This study \\
\hline \multirow[t]{6}{*}{ Calcium ( $\mathrm{mg} \mathrm{dL}^{-1}$ ) } & California Sea Lion/Zalophus californianus & $9.4 \pm 0.5$ & Roletto 1993 \\
\hline & Pacific Harbour Seal/Phoca vitulina richardii & $9.7 \pm 0.5$ & Roletto 1993 \\
\hline & Harbor seal/Phoca vitulina & $9.9 \pm 1.4$ & Trumble \& Castellini 2002 \\
\hline & South American sea lion/Otaria flavescens (July 2009-April 2011) & $10.1 \pm 2.9$ & This study \\
\hline & Northern elephant seal/Mirounga angustirostris & $10.4 \pm 0.6$ & Roletto 1993 \\
\hline & Gray whale/Eschrichtius robustus & $13.7 \pm$ n.d. & Dailey et al. 2000 \\
\hline \multirow[t]{3}{*}{ Lactate $\left(\mathrm{mg} \mathrm{dL}^{-1}\right)$} & Harbor seal/Phoca vitulina & $3.96 \pm 1.6$ & Davis 1983 \\
\hline & Harbor seal /Phoca vitulina & $7.2 \pm$ n.d. & Davis et al. 1991 \\
\hline & South American sea lion/Otaria flavescens (July 2009-April 2011) & $50.7 \pm 23.3$ & This study \\
\hline
\end{tabular}

n.d.= no data 
Table 3 continued / Continuación Tabla 3

\begin{tabular}{|c|c|c|c|}
\hline Parameter & Species & Value & Reference \\
\hline \multirow[t]{11}{*}{ Glucose $\left(\mathrm{mg} \mathrm{dL}^{-1}\right)$} & Gray whale/Eschrichtius robustus & $14 \pm$ n. d. & Dailey et al. 2000 \\
\hline & Amazonian manatee/Trichechus inunguis & $34.4 \pm 2.1$ & Colares et al. 2000 \\
\hline & Short-beaked common dolphins/Delphinus delphis & $94 \pm 22.4$ & Ortiz \& Worthy 2000 \\
\hline & Harbor seal/Phoca vitulina & $102.6 \pm 10.8$ & Davis 1983 \\
\hline & Weddellseal/Leptonychotes weddellii & $108.1 \pm 10.9$ & Sakamoto et al. 2009 \\
\hline & California Sea Lion/Zalophus californianus & $113 \pm 30$ & Roletto 1993 \\
\hline & South American sea lion/Otaria flavescens (July 2009-April 2011) & $130.2 \pm 26.8$ & This study \\
\hline & Pacific Harbour Seal/Phoca vitulina richardii & $136 \pm 25$ & Roletto 1993 \\
\hline & Northern elephant seal/Mirounga angustirostris & $144 \pm 27$ & Roletto 1993 \\
\hline & Harbor seal/Phoca vitulina & $154.8 \pm$ n.d. & Davis et al. 1991 \\
\hline & Harbor seal/Phoca vitulina & $165.9 \pm 25.8$ & Trumble \& Castellini 2002 \\
\hline \multirow[t]{4}{*}{ Total cholesterol $\left(\mathrm{mg} \mathrm{dL}^{-1}\right)$} & Harbor seal/Phoca vitulina & $337.2 \pm 85.3$ & Trumble \& Castellini 2002 \\
\hline & Gray whale/Eschrichtius robustus & $339 \pm$ n.d. & Dailey et al. 2000 \\
\hline & Weddellseal/Leptonychotes weddellii & $368 \pm 97$ & Sakamoto et al. 2009 \\
\hline & South American sea lion/Otaria flavescens (July 2009-April 2011) & $521.0 \pm 448.2$ & This study \\
\hline \multirow[t]{6}{*}{$\mathrm{LDH}\left(\mathrm{IU} \mathrm{L}^{-1}\right)$} & South American sea lion/Otaria flavescens (July 2009-April 2011) & $280 \pm 0.1$ & This study \\
\hline & Gray whale/Eschrichtius robustus & $409 \pm$ n.d. & Dailey et al. 2000 \\
\hline & Northern elephant seal/Mirounga angustirostris & $556 \pm 294$ & Roletto 1993 \\
\hline & Pacific Harbour Seal/Phoca vitulina richardii & $811 \pm 265$ & Roletto 1993 \\
\hline & California Sea Lion/Zalophus californianus & $888 \pm 343$ & Roletto 1993 \\
\hline & Harbor seal/Phoca vitulina & $3345 \pm 1258$ & Trumble \& Castellini 2002 \\
\hline \multirow[t]{2}{*}{ Cholinesterase $\left(\mathrm{IU} \mathrm{L}^{-1}\right)$} & South American sea lion/Otaria flavescens (July 2009-April 2011) & $665.9 \pm 238.6$ & This study \\
\hline & California Sea Lion/Zalophus californianus & $815,6 \pm 125.6$ & Donovan et al. 1994 \\
\hline Lysozyme (IU mL $\left.L^{-1}\right)$ & South American sea lion/Otaria flavescens (July 2009-April 2011) & $119.1 \pm 65.4$ & This study \\
\hline Inh.Ant.Cap. $(\%)$ & South American sea lion/Otaria flavescens (July 2009-April 2011) & $96.6 \pm 2.3$ & This study \\
\hline \multirow[t]{5}{*}{$\operatorname{Urea}\left(\mathrm{mg} \mathrm{dL}^{-1}\right)$} & Weddellseal/Leptonychotes weddellii & $25.9 \pm 13.7$ & Sakamoto et al. 2009 \\
\hline & Amazonian manatee/Trichechus inunguis & $26.6 \pm 2.6$ & Colares et al. 2000 \\
\hline & Harbor seal/Phoca vitulina & $34.9 \pm 9.6$ & Trumble \& Castellini 2002 \\
\hline & Gray whale/Eschrichtius robustus & $58 \pm$ n.d. & Dailey et al. 2000 \\
\hline & South American sea lion/Otaria flavescens (July 2009-April 2011) & $90.3 \pm 20.7$ & This study \\
\hline \multirow[t]{3}{*}{$\operatorname{AST}\left(\mathrm{IU} \mathrm{L} \mathrm{L}^{-1}\right)$} & South American sea lion/Otaria flavescens (July 2009-April 2011) & $20.6 \pm 10.9$ & This study \\
\hline & Gray whale/Eschrichtius robustus & $27.9 \pm$ n.d. & Dailey et al. 2000 \\
\hline & Harbor seal/Phoca vitulina & $75.2 \pm 29.9$ & Trumble \& Castellini 2002 \\
\hline \multirow[t]{3}{*}{$\operatorname{ALT}\left(\mathrm{IU} \mathrm{L} \mathrm{L}^{-1}\right)$} & South American sea lion/Otaria flavescens (July 2009-April 2011) & $14.0 \pm 9.0$ & This study \\
\hline & Gray whale/Eschrichtius robustus & $20 \pm$ n.d. & Dailey et al. 2000 \\
\hline & Harbor seal/Phoca vitulina & $24.6 \pm 11.7$ & Trumble \& Castellini 2002 \\
\hline \multirow[t]{2}{*}{ AST/ALT } & South American sea lion/Otaria flavescens (Julio 2009-Abril 2011) & $5.7 \pm 10.9$ & This study \\
\hline & Gray whale/Eschrichtius robustus & 13.95 & Dailey et al. 2000 \\
\hline \multirow[t]{2}{*}{$\mathrm{PMN}(\%)$} & South American sea lion/Otaria flavescens (July 2009-April 2011) & $51.1 \pm 5.3$ & This study \\
\hline & Harbor seal/Phoca vitulina & $60.2 \pm 10.4$ & Trumble \& Castellini 2002 \\
\hline \multirow[t]{2}{*}{ Lymphocytes (\%) } & Harbor seal/Phoca vitulina & $28.1 \pm 9.7$ & Trumble \& Castellini 2002 \\
\hline & South American sea lion/Otaria flavescens (July 2009-April 2011) & $35.1 \pm 5.5$ & This study \\
\hline \multirow[t]{2}{*}{ Eosinophils (\%) } & Harbor seal/Phoca vitulina & $3.2 \pm 2.9$ & Trumble \& Castellini 2002 \\
\hline & South American sea lion/Otaria flavescens (July 2009-April 2011) & $8.0 \pm 3.7$ & This study \\
\hline \multirow[t]{2}{*}{ Monocytes (\%) } & South American sea lion/Otaria flavescens (July 2009-April 2011) & $4.8 \pm 3.0$ & This study \\
\hline & Harbor seal/Phoca vitulina & $8.9 \pm 5.3$ & Trumble \& Castellini 2002 \\
\hline \multirow[t]{2}{*}{ Basophils (\%) } & South American sea lion/Otaria flavescens (July 2009-April 2011) & $0.6 \pm 0.4$ & This study \\
\hline & Harbor seal/Phoca vitulina & $0.9 \pm 1.1$ & Trumble \& Castellini 2002 \\
\hline Leukocyte bacilliforms (\%) & South American sea lion/Otaria flavescens (July 2009-April 2011) & $0.3 \pm 0.4$ & This study \\
\hline
\end{tabular}

n.d. = no data 
against microbial invasion. It is present in mucus, secretions, saliva and plasma, and has been utilized as an innate immune response indicator in fish (Callewaert \& Michiels 2010, Saurabh \& Sahoo 2008). In marine mammals, there are only a few reports of immune response, and skin lysozyme presence has only been reported in $P$. vitulina and Callorhinus ursinus (Meyer et al. 2003). The importance of these enzymes lies in the presence of microorganisms in the marine environment, which are capable of causing skin lesions, such as dermatitis due to fungi in Californian sea lions (Guillot et al. 1998). In this study, we present new data on the plasmatic activity of lysozyme in $O$. flavescens individuals, which did not have any apparent skin lesions when they were captured. Changes in lysozyme activity could be associated with the occurrence of pathogens in mammals (Maraghi et al. 2012); in fish (Saurabh \& Sahoo 2008), mammals and other animals (Demers \& Bayne 1997, Callewaert \& Michiels 2010), changes in activity have also been linked to stressinducing environmental conditions, pollution and seasonal variability.

The diagnostic value of determining the plasma activity of cholinesterase is that it can be inhibited by organophosphorus and carbamate plagicides, as well as by heavy metals and detergents (Guilhermino et al. 1998). Therefore, a drop in the activity of this enzyme suggests a degree of adsorption and exposure to some substance that inhibits its activity. Average plasma cholinesterase activity found for captured and rehabilitated Californian sea lions (Donovan \& Zinkl 1994; Table 3) was greater than that observed in the plasma during the first sampling period and very close to the activity measured during the second sampling period (Table 1), with a significant difference between both study periods. This may indicate the presence of some inhibitor of this enzyme in the environment during the first sampling period, however, the number of samples (3) in the study by Donovan \& Zinkl (1994) and the difference in focal species limits comparison with the present study. Nevertheless, during the first sampling period, the activity of cholinesterase ranged between 131.6 and $636.1 \mathrm{IU} \mathrm{L}^{-1}$. The individual that displayed lowest cholinesterase activity (131.6 $\left.\mathrm{IU} \mathrm{L}^{-1}\right)$ also had the highest plasma cholesterol concentration (1552.5 $\left.\mathrm{mg} \mathrm{dL}^{-1}\right)$. The ratio of cholesterol/cholinesterase of this individual (male), with a body length close to $143 \mathrm{~cm}$, seems to be altered compared to other individuals (Fig. 2). This result could indicate a certain level of exposure to some substance with the same characteristics as anticholinesterase. Further study is required in order to better interpret this result, given the high daily mobility of sea lions in their environment, up to $154 \mathrm{~km}$ distance from the coast (Hückstädt \& Krautz 2004). Hypothetically, sea lions might eat at one contaminated site, located far away from the breeding colony. Around the Nature Sanctuary of Islote Lobería de Cobquecura there is intensive agricultural land-use where pesticides are used, and which via weathering can be transported to the coastal environment and bioaccumulate in the trophic web.

The average concentration of phosphorus found in individuals sampled from the Cobquecura breeding colony (Table 1) was within the same range and is comparable to that reported for other marine mammals (Table 3). The composition of elements in the blood, such as phosphorus, can vary per individual due to diverse factors, such as life stage, sex, and reproductive status (Sterner \& Elser 2002). Moreover, phosphorus is present in the structure of important biomolecules, such as nucleic acids (Sterner \& Elser 2002) and the skeleton (Diem 1970).

In this study, the differential blood cell counts obtained for $O$. flavescens revealed that most of the circulating white blood cells were polymorphonuclear neutrophils $(\mathrm{PMN})$ and lymphocytes, which is consistent with the normal blood cell count of mammals, including humans (Costa et al. 1993). However, the results of the blood cell count reported here also reveal high eosinophilia during the first sampling period, and are significantly different from values during the second period. Values from the first sampling period were also higher than the average percentage of eosinophils observed in Californian sea lions, including clinically healthy and parasite infected animals (Roletto 1993). In the latter study, the range of quantified eosinophils was higher for individuals with parasites $(0-12 \%)$ compared with those that were clinically healthy $(0-8 \%)$. Eosinophilia in mammals also has been associated with parasitism (Noemi \& Atias 1987, Rodríguez et al. 2000, Urquhart et al. 2001, Medina et al. 2002, Mussart \& Coppo 2009). In marine mammals, the existence of skin, lung, stomach, and intestine parasites is widely known (e.g., Carvajal et al. 1983, Fernández 1987, Roletto 1993, Dailey et al. 2000). For O. flavescens, intestinal parasites have been reported (Dailey \& Brownell 1972, Cattan et al. 1976, George-Nascimento \& Carvajal 1981, George-Nascimento \& Urrutia 2000), and are acquired during immature developmental stages through ingestion of food (George-Nascimento \& Llanos 1995). The high concentration of globulins found during the first sampling period may also be explained by an immune response due to parasitism, which would be consistent with the presence of eosinophilia. 
Depending on the type of parasitic tissue, there may be a greater or lesser degree of cell destruction. An indicator of tissue destruction is the enzyme activity of lactate dehydrogenase, or $\mathrm{LDH}$, which is found in the cells of tissues, which upon suffering a lesion; liberate this enzyme into the blood stream (Dufour et al. 2005). In this study, LDH displayed low plasma activity, indicating that there was no abnormal tissue damage. In spite of this, eosinophilia detected suggests that parasitism in Otaria flavescens may be present. In O. flavescens, parasites spend most of their life cycle within the digestive tract (Dailey \& Brownell 1972, Cattan et al. 1976, George-Nascimento \& Carvajal 1981, George-Nascimento \& Urrutia 2000). Intestinal parasites, although able to migrate through other organs (e.g., Lungs in the Loos cycle; Steeger \& Vargas 1960), produce less damage than parasites that occupy the liver, for example, as their final organ (Carrada-Bravo 2007), or skeletal muscles (Gottstein et al. 2009), among others.

Finally, to characterize the health of Otaria a larger sample size is needed to conduct a more robust statistical data analysis. The small sample size in this study is insufficient for exploring the complete range of analytes measured in individuals that inhabit the breeding colony. It is nevertheless useful as seminal information for comparing and contrasting with information obtained in the future on the blood chemistry and hematology of this species and therefore, makes a contribution to species conservation in the Southeast Pacific.

\section{ACKNOWLEDGMenTs}

This study is part of the Programa de Investigación Marina de Excelencia (PIMEX) of the Faculty of Natural and Oceanographic Sciences (Universidad de Concepción, Chile) funded by Celulosa Arauco y Constitución S.A. R. González received additional funding from Programa Copas Sur-Austral (PFB-31). We acknowledge Pablo Carrasco, Danilo Alegría, and Omar Muñoz for their help in the field.

\section{LITERATURE CITED}

Aguayo-Lobo A, H Díaz, J Yáñez, F Palma \& M Sepúlveda. 1998. Censo poblacional del lobo marino común en el litoral de la V a la IX Regiones. Informe Final Proyecto FIP 9651: 1-251. Doppler Ltda, Valparaíso. <http://www.fip.cl/ Archivos/Hitos/Informes/inffinal\%2096-51.pdf>

Aguayo A \& R Maturana. 1973. Presencia del lobo marino común Otaria flavescens, en el litoral chileno. Arica $\left(18^{\circ} 27^{\prime}\right.$ S) a punta Maiquillahue (39²7' W). Biología Pesquera 6: 45-75.
Bartheld J, H Pavés, F Contreras, C Vera, C Manque, D Miranda, D Sepúlveda, P Artacho \& L Ossman. 2008. Cuantificación poblacional de lobos marinos en el litoral de la I a IV Región. Informe Final Proyecto FIP N²006-50: 1-124. <http://www.fip.cl/Archivos/Hitos/Informes/ inffinal\%202006-50.pdf>

Bastida R \& D Rodríguez. 2003. Mamíferos marinos de Patagonia y Antártida, 208 pp. Vazquez Manzini, Buenos Aires.

Bossart GD, T Reidarson, LA Dierauf \& DA Duffield. 2001. Clinical pathology. In: Dierauf LA \& FMD Gulland (eds). Handbook of marine mammal medicine, pp. 383-436. CRC Press, Boca Raton.

Butler PJ \& DR Jones. 1997. Physiology of diving of birds and mammals. Physiological Reviews 77(3): 837-894.

Callewaert L \& CW Michiels. 2010. Lysozymes in the animal kingdom. Journal of Biosciences 35(1): 127-160.

Cappozzo HL \& WF Perrin. 2009. South American sea lion Otaria avescens. In: Perrin WF, B Wursig \& JGM Thewissen (eds). Encyclopedia of marine mammals, pp. 1076-1079. AcademicPress/Elsevier, Amsterdam.

Carrada-Bravo T. 2007. Fasciola hepatica: Ciclo biológico y potencial biótico. Revista Mexicana de Patología Clínica 54(1): 21-27.

Carvajal J, LE Durán \& M George-Nascimento. 1983. Ogmogaster heptalineatus $\mathrm{n}$. sp. (Trematoda: Notocotylidae) from the Chilean sea lion Otaria flavescens. Systematic Parasitology 5(3): 169-173.

Cattan P, B Babero \& D Torres. 1976. The helminth fauna of Chile: IV. Nematodes of the genera Anisakis Dujardln, 1845 and Phocanema Myers, 1954 in relation with gastric ulcers in a South American Sea Lion, Otaria byronia. Journal of Wildlife Diseases 12(4): 511-515.

Colares E, I Colares, A Bianchini \& E Santos. 2000. Seasonal variations in blood parameters of the Amazonian Manatee, Trichechus inunguis. Brazilian Archives of Biology and Technology 43(2): 165-171.

Costa J, J Madrid \& S Zamora. 1993. Manual de clases prácticas de fisiología animal, $236 \mathrm{pp}$. Universidad de Murcia, Murcia.

Crespo E, S Pedraza, S Dans, M Koen-Alonso, L Reyes, N García \& M Coscarella. 1997. Direct and indirect effects of the high seas fisheries on the marine mammal populations in the northern and central Patagonian coast. Journal of Northwest Atlantic Fishery Science 22: 189-207.

Dailey M \& R Brownell. 1972. A checklist of marine mammal parasites. In: Ridgway SH (ed). Mammals of the sea: biology and medicine, pp. 528-589. Charles C Thomas, Springfield.

Dailey M, FM Gulland, LJ Lowenstine, P Silvagni \& D Howard. 2000. Prey, parasites and pathology associated with the mortality of a juvenile gray whale (Eschrichtius robustus) stranded along the northern California coast. Diseases of Aquatic Organisms 42(2): 111-117. 
Dangerfield W, R Finlayson, G Myatt \& M Mead. 1976. Serum lipoproteins and atherosclerosis in animals. Atherosclerosis 25(1): 95-106.

Dans S, W Sielfeld, A Aguayo, G Giardino \& MA Mandiola. 2012. Estado y tendencia de las poblaciones. En: Crespo E, D Oliva, S Dans \& M Sepúlveda (eds). Estado de situación del lobo marino común en su área de distribución, pp. 20-37. Editorial Universidad de Valparaíso, Valparaíso.

Davis RW. 1983. Lactate and glucose metabolism in the resting and diving harbor seal (Phoca vitulina). Journal of Comparative Physiology B 153(2): 275-288.

Davis RW, M Castellini, T Williams \& G Kooyman. 1991. Fuel homeostasis in the harbor seal during submerged swimming. Journal of Comparative Physiology B 160(6): 627-635.

De la Torriente A, R Quiñones, D Miranda-Urbina \& F Echevarría. 2010. South American sea lion and spiny dogfish predation on artisanal catches of southern hake in fjords of Chilean Patagonia. ICES Journal of Marine Science 67(2): 294-303.

Demers NE \& CJ Bayne. 1997. The immediate effects of stress on hormones and plasma lysozyme in rainbow trout. Developmental \& Comparative Immunology 21(4): 363-373.

Diem K. 1970. Documenta Geigy, 535 pp. Geigy Pharmaceuticals, Ardsley.

Donovan D \& J Zinkl. 1994. Modifications of a cholinesterase method for determination of erythrocyte cholinesterase activity in wild mammals. Journal of Wildlife Diseases 30(2): 234-240.

Dufour D, J Lott \& J Henry. 2005. Enzimología clínica. En: Henry JB (ed). El laboratorio en el diagnóstico clínico, pp. 294-296. Marbán Libros, Madrid.

Ellis AE. 1990a. Lysozyme assays. In: Stolen JS, TC Fletcher, DP Anderson, BS Roberson \& WB Van Muiswinkel (eds). Techniques in fish immunology 1: 101-103. SOS Publications, Fair Haven.

Ellis AE. 1990b. Serum antiproteases in fish. In: Stolen JS, TC Fletcher, DP Anderson, BS Roberson \& WB van Muiswinkel (eds). Techniques in fish immunology 1: 9599. SOS Publications, Fair Haven.

Fernández J. 1987. Nuevos registros de parásitos en mamíferos marinos chilenos. Parasitología al Día 11(3): 120-126.

Gales N \& R Mattlin. 1998. Fast, safe, field-portable gas anesthesiaforotariids. Marine Mammal Science 14(2): 355-361.

George-Nascimento M \& J Carvajal. 1981. Helmintos parásitos del lobo marino común Otaria flavescens en el Golfo de Arauco, Chile. Boletín Chileno de Parasitología 36(3): $72-73$.

George-Nascimento M \& A Llanos. 1995. Microevolutionary implications of allozymic and morphometric variations in sealworms Pseudoterranova sp. (Ascaridoidea: Anisakidae) among sympatric hosts from the Southeastern Pacific Ocean. International Journal for Parasitology 25(10): 1163-1171.
George-Nascimento M \& X Urrutia. 2000. Pseudoterranova cattani sp. n. (Nematoda, Ascaridoidea, Anisakidae), a gastric parasite of South American sea lion Otaria byronia De Blainville in Chile. Revista Chilena de Historia Natural 73(1): 93-98.

George-Nascimento M, R Bustamante \& C Oyarzún. 1985. Feeding ecology of the South sea lion Otaria flavescens: Food contents and food selectivity. Marine Ecology Progress Series 21: 135-143.

Gottstein B, E Pozio \& K Nöckler. 2009. Epidemiology, diagnosis, treatment, and control of trichinellosis. Clinical Microbiol Reviews 22(1): 127-145.

Guilhermino L, P Barros, M Silva \& AMVM Soares. 1998. Should the use of inhibition of cholinesterases as a specific biomarker for organophosphate and carbamate pesticides be questioned. Biomarkers 3(1): 157-163.

Guillot J, T Petit, F Degorce-Rubiales, E Gueho \& R Chermette. 1998. Dermatitis caused by Malassezia pachydermatis in a California sea lion (Zalophus californianus). The Veterinary Record 142(12): 311-312.

Halloran D \& A Pearson. 1972. Blood chemistry of the brown bear (Ursus arctos) from southwestern Yukon territory, Canada. Canadian Journal of Zoology 50(6): 827-833.

Hochachka P \& G Somero. 1973. Strategies of biochemical adaptation, 358 pp. W. B. Saunders Company, Philadelphia.

Hückstädt LA \& T Antezana. 2006. The diet of Otaria flavescens in Chile: what do we know? In: Trites A, S Atkinson, D DeMaster, L Fritz, T Gelatt, L Rea \& K Wynne (eds). Sea lions of the world, pp. 83-97. Alaska Sea Grant College Program, University of Alaska Fairbanks, Fairbanks.

Hückstädt L \& MC Krautz. 2004. Interaction between southern sea lions Otaria flavescens and jack mackerel Trachurus symmetricus commercial fishery off Central Chile: a geostatistical approach. Marine Ecology Progress Series 282: 285-294.

Hückstädt LA, CP Rojas \& T Antezana. 2007. Stable isotope analysis reveals pelagic foraging by the Southern sea lion in central Chile. Journal of Experimental Marine Biology and Ecology 347: 123-133.

Hyvarinen A, T Helle, R Vayrynen \& P Vayrynen. 1975. Seasonal and nutritional effects on serum proteins and urea concentration in the reindeer (Rangifer tarandus L.). British Journal of Nutrition 33: 63-72.

Ikehara T, Y Eguchi, S Kayo \& H Takei. 1996. Amino acid sequences of hemoglobin â chains of five species of pinnipeds: Neophoca cinerea, Otaria byronia,Eumetopias jubatus, Pusa hispida, and Pagophilus groenlandica. Journal of Protein Chemistry 15(7): 659-665.

King J. 1983. Seals of the world, 240 pp. Oxford University Press, Cambridge.

Koen-Alonso M, E Crespo, S Pedraza, N García \& M Coscarella. 2000. Food habits of the South American sea lion, Otaria flavescens, off Patagonia, Argentina. Fishery Bulletin 98: 250-263. 
Koopman H, A Westgate, A Read \& D Gaskin. 1995. Blood chemistry of wild harbor porpoises Phocoema phocoema (L.). Marine Mammal Science 11: 123-135.

Krautz MC, LR Castro, M González, JC Vera \& HE González. 2012. Concentration of ascorbic acid and innate immune effectors in Engraulis ringens and Strangomera bentincki during their main spawning period (2007-2008) in the Humboldt Current system off Chile. Marine Biology 159(2): 303-317.

Lander ME, BS Fadely, TS Gelatt, LD Rea \& TR Loughlin. 2014. Serum chemistry reference ranges for Steller Sea Lion (Eumetopias jubatus) Pups from Alaska: Stock differentiation and comparisons within a North Pacific Sentinel Species. EcoHealth 10: 376-393.

Lehninger A, D Nelson \& M Cox. 2001. Principios de bioquímica, 1264 pp. Ediciones Omega, Barcelona.

LeResche R, U Seal, P Karns \& A Franzmann. 1974. A review of blood chemistry of moose and other cervidae with emphasis on nutritional assessment. Le Naturaliste Canadien 101: 263-290.

Maraghi S, DH Molyneux \& KR Wallbanks. 2012. Lysozyme activity in the plasma of rodents infected with their homologous trypanosomes. Iranian Journal of Parasitology 7(4): 86-90.

Matula GJ, J Lindzey \& H Rothenbacher. 1980. Sex, age, and seasonal differences in the blood profile of black bears captured in northeastern Pennsylvania. International Conference on Bear Research and Management 4: 49-56.

Medina J, M Tantaleán, M León \& M Cano. 2002. Diphyllobothrium pacificum en niños del Perú. Diagnóstico, Perú 41(4): 161-164.

Meyer M, U Seegers, J Herrmann \& A Schnapper. 2003. Further aspects of the general antimicrobial properties of pinniped skin secretions. Diseases of Aquatic Organisms 53: 177-179.

Mussart N \& J Coppo. 2009. Indicadores sanguíneos de daño hepático en novillos cruza cebú parasitados por Fasciola hepatica. Revista Veterinaria 20(2): 81-85.

Muñoz L, G Pavez, R Quiñones, D Oliva, M Santos \& M Sepúlveda. 2013. Diet plasticity of the South American sea lion in Chile: stable isotope evidence. Revista de Biología Marina y Oceanografía 48(3): 613-622.

Noemi I \& A Atias. 1987. Eosinofilia y parasitosis. Parasitología Clínica 57: 457-461.

Oliva D, M Sepúlveda, LR Durán, A Urra, W Sielfeld, R Moraga, G Pavés \& L Muñoz. 2012. Cuantificación poblacional de lobos marinos en las Regiones X-XI y propuesta de escenarios de manejo. Informe Final Proyecto, Fondo de Administración Pesquero, FAP ID 4728-46-LP11: $1-100$.

Oporto J, L Brieva, R Navarro \& A Turner. 1999. Cuantificación poblacional de lobos marinos en el litoral de la X y XI Regiones. Informe Técnico, Fondo de Investigación Pesquera FIP-IT/97-44: 1-237. <http:// www.fip.cl/Archivos/Hitos/Informes/inffinal\%209744.pdf>
Ortiz R \& G Worthy. 2000. Effects of capture on adrenal steroid and vasopressin concentrations in free-ranging bottlenose dolphins (Tursiops truncatus). Comparative Biochemistry and Physiology A 125: 317-324.

Osgood W. 1943. The mammals of Chile. Publication Field Museum of Natural History (Zoology) Series 30: 100-101.

Panfoli I, B Burlando \& A Viarengo. 1999. Cyclic ADPribose-dependant $\mathrm{Ca} 2+$ release is modulated by free $[\mathrm{Ca} 2+]$ in the Scallop Sarcoplasmic reticulum. Biochemical and Biophysical Research Communications 257: 57-62.

Riedman M. 1990. The pinnipeds: Seals, sea lions, and walruses, 439 pp. University of California Press, Berkeley.

Rodríguez R, L Torrado, T Rivero \& F Xavier. 2000. La fasciolasis hepática humana en Camaguey. Revista Electrónica ‘Archivo Médico de Camagüey’ 4(2): 6-11.

Roletto J. 1993. Hematology and serum chemistry values for clinically healthy and sick pinnipeds. Journal of Zoo and Wildlife Medicine 24(2): 145-157.

Rosas FCW, MC Pinedo, M Marmontel \& M Haimovici. 1994. Seasonal movements of the South American sea lion (Otaria flavescens, Shaw) off the Rio Grande do Sul coast, Brazil. Mammalia 58: 51-59.

Sakamoto K, K Sato, Y Naito, Y Habara, M Ishizuka \& S Fujita. 2009. Morphological features and blood parameters of Weddell seal (Leptonychotes weddellii) mothers and pups during the breeding season. Japanese Society of Veterinary Science 71(3): 341-344.

Saurabh S \& PK Sahoo. 2008. Lysozyme: an important defense molecule of fish innate immune system. Aquaculture Research 39: 223-239.

Scheffer V. 1958. Seals, Sea Lions and Walruses. A review of the Pinnipedia, 179 pp. Stanford University Press, Stanford.

Schiedek D. 1997. Marenzelleria viridis (Verril, 1873) (Polychaeta), a new benthic species within European coastal waters. Some metabolic features. Journal of Experimental Marine Biology and Ecology 211: 85-101.

Schlatter R \& G Riveros. 1997. Historia natural del Archipiélago Diego Ramírez, Chile. Serie Científica INACH 47: 87-112.

Seal U, L Mech \& V Ballenberche. 1975. Blood analyses of wolf pups and their ecological and metabolic interpretation. Journal of Mammalogy 56: 64-75.

Sepúlveda M, H Ochoa-Acuña \& B Homer. 1999. Age-related changes in hematocrito, hemoglobin and plasma protein in Juan Fernández fur seals (Arctocephalus philippii). Marine Mammals Science 15(2): 575-581.

Sepúlveda M, M Pérez, W Sielfeld, D Oliva, L Durán, L Rodríguez, V Araos \& M Buscaglia. 2007. Operational interaction between South American sea lions Otaria flavescens and artisanal (small-scale) fishing in Chile: results from interview surveys and on-board observations. Fisheries Research 83: 332-340. 
Sepúlveda M, D Oliva, A Urra, P Pérez-Álvarez, R Moraga, D Schrader, P Inostroza, A Melo, H Díaz \& W Sielfeld. 2011. Distribution and abundance of the South American sea lion Otaria flavescens (Carnivora: Otariidae) along the central coast off Chile. Revista Chilena de Historia Natural 84: 97-106.

Sielfeld W, C Guerra, R Durán, E Acuña, A Aguayo-Lobo, M Sepúlveda, F Palma, A Malinarich, G Cerda, A Bolvaran, R Grau, X Veloso, Y Guerra, M Vargas, N Amado, R Peredo \& J Galaz. 1997. Monitoreo de la pesquería y censo del lobo marino común en el litoral de la I-IV Regiones. Informe Técnico, Fondo de Investigación Pesquera FIP-IT/95-28: 1-105. <http://www.fip.cl/ Archivos/Hitos/Informes/inffinal\%2095-28.pdf>

Steeger A \& L Vargas. 1960. Erupción reptante: Dermatosis producida por larva de Ancylostoma caninum. Revista Chilena de Pediatría 31(10): 511-521.

Sterner R \& J Elser. 2002. Ecological stoichiometry: The biology of elements from molecules to the biosphere, 584 pp. Princeton University Press, Princeton.

Trumble S \& M Castellini. 2002. Blood chemistry, hematology and morphology of wild harbor seal pups in Alaska. Journal of Wildlife Management 66(4): 1197-1207.

Urich K. 1994. Comparative animal biochemistry, 782 pp Springer-Verlag Berlin, Heidelberg.

Urquhart G, J Armour, A Duncan \& F Jennings. 2001. Parasitología veterinaria, 368 pp. Acribia, Zaragoza.
Vaz-Ferreira R. 1982. Otaria flavescens (Shaw), South American sea lion. In: Mammals in the Seas: Small cetaceans, seals, sirenians and otters. FAO Fisheries Series 5(4): 477 495.

Venegas C, J Gibbons, A Aguayo, W Sielfeld, J Acevedo, N Amado, J Capella, G Guzmán \& C Valenzuela. 2001. Cuantificación poblacional de los lobos marinos en la XII Región. Fondo de Investigación Pesquera Proyecto FIP $\mathrm{N}^{\circ}$ 2000-22: 1-92. <http://www.fip.cl/Archivos/Hitos/ Informes/inffinal\%202000-22.pdf $>$.

Werner R \& C Campagna. 1995. Diving behaviour of lactating southern sea lions (Otaria flavescens) in Patagonia. Canadian Journal of Zoology 73: 1975-1982.

Yuan A, CH Siu \& CP Chia. 2001. Calcium requirement for efficient phagocytosis by Dictotelium discoideum. Cell Calcium 29(4): 229-238.

Zuo X \& PTK Woo. 1997. In vivo neutralization of Criptobias salmositica metallo-protease by á 2-macroglobulin in the blood of rainbow trout Oncorhynchus mykiss and in brook charr Salvelinus fontinalis. Diseases of Aquatic Organisms 29: 67-72.

Received 15 November 2013 and accepted 17 December 2014

Associated Editor: Maritza Sepúlveda M. 